\section{Una experiencia de extensión universitaria que desafía la distancia entre teoría y práctica: construir saberes entre docentes, referentes comunitarios y estudiantes}

\author{
Claudia Bang \\ claudiabang@yahoo.com.ar \\ Chiara Elena Barile \\ chiarabarile@gmail.com
}

Marina Guldris

guldrismarina@gmail.com

\author{
Federico Chaves \\ chaves_federico@hotmail.com \\ Universidad de Buenos Aires, \\ Argentina.
}

Investigación y extensión universitaria / Intervenciones
RECEPCIÓN: 29/06/18

ACEPTACIÓN FINAL: 20/09/18

\section{Resumen}

El objetivo de este escrito es describir y analizar los aportes e implicancias de la inclusión de prácticas comunitarias en la formación universitaria a partir de una experiencia de extensión desarrollada en la Facultad de Psicología de la Universidad de Buenos Aires (UBA), y su articulación a procesos de investigación y docencia. Partiendo de breves viñetas, abordamos los atravesamientos subjetivos de esta experiencia y cuestionamos la construcción de roles profesionales rígidos y estáticos, así como una formación universitaria centrada en la relación asimétrica y distante entre docente-estudiante, alejada de las problemáticas sociocomunitarias concretas. Se concluye que uno de los principales aportes de esta experiencia está en la creación colectiva de una posición reflexiva, implicada y activa, con relación a las problemáticas psicosociales. Consideramos que este proceso aporta en la construcción de un puente entre universidad y realidad social, el que esperamos fortalecer a partir de articulaciones múltiples entre prácticas de extensión e investigación participativa.

Palabras clave: extensión universitaria, experiencia, formación profesional, investigación participativa, comunidad.
An experience of university extension that challenges the distance between theory and practice: building knowledge among teachers, community leaders and students

\section{Abstract}

The objective of this paper is to describe and analyze the contributions and implications of the inclusion of community practices in university education, based on an extension experience developed at the School of Psychology of the University of Buenos Aires, and its articulation to processes of research and teaching Starting from brief vignettes, we address the subjective traversal of this experience, questioning the construction of rigid and static professional roles, as well as a university education focused on the asymmetric and distant relationship between teacher-student, away from the specific sociocommunity problems. It is concluded that one of the main contributions of this experience is in the collective creation of a reflective, involved and active position in relation to psycho-social problems. We consider that this process contributes to the construction of a bridge between university and social reality, which we hope to strengthen based on multiple articulations between extension practices and participatory research.

Keywords: university extension, experience, professional training, participatory research, community.

\begin{abstract}
Uma experiência de extensão universitária que desafia a distância entre teoria e prática: construir conhecimento entre professores, líderes comunitários e estudantes
\end{abstract}

\section{Resumo}

O objetivo deste artigo é descrever e analisar as contribuições e implicações da inclusão de práticas comunitárias no ensino universitário, a partir de uma experiência de extensão universitária desenvolvida na Faculdade de Psicologia da Universidade de Buenos Aires, e sua articulação aos processos de pesquisa e ensino. Com base na análise de vinhetas breves, abordamos os atravessamentos subjetivos desta experiência, questionando a construção de papéis profissionais rígidos e estáticos, bem como uma formação universitária focada na relação assimétrica e distante entre professor-aluno, longe dos problemas sociocomunitários concretos. Conclui-se que uma das principais contribuições dessa experiência está na criação coletiva de uma posição reflexiva, comprometida e ativa em relação aos problemas psicossociais. Consideramos que esse processo contribui para a construção de uma ponte entre a universidade e realidade social, que esperamos fortalecer a partir de múltiplas articulações entre práticas de extensão e pesquisa participativa.

Palavras-chave: extensão universitária, experiência, formação profissional, pesquisa participativa, comunidade.

Para citación de este artículo: Bang, C.; Barile, C.; Guldris, M. y Chaves, F. (2018). Una experiencia de extensión universitaria que desafía la distancia entre teoría y práctica: construir saberes entre docentes, referentes comunitarios y estudiantes. +E: Revista de Extensión Universitaria, 8(9), julio-diciembre, 167-182. doi: 10.14409/extension.v8i9.Jul-Dic.7854. 


\author{
"La universidad será democrática si sabe usar \\ su saber hegemónico para recuperar y hacer \\ posible el desarrollo autónomo de los saberes \\ no hegemónicos, manejados en las prácticas \\ de las clases sociales oprimidas y de los grupos \\ o estratos socialmente discriminados." \\ (Sousa Santos, 1998:278)
}

\section{Introducción}

Este escrito nace de la experiencia de un proyecto de extensión universitaria en la Facultad de Psicología de la Universidad de Buenos Aires (UBA). Se trata del Voluntariado Universitario presentado por la Cátedra de Salud Pública/Salud Mental II "Arte, juego y salud mental”, cuyo objetivo es participar en las prácticas comunitarias de la red interinstitucional RIOBA (Red de Instituciones de Once, Balvanera y Abasto) y producir colectivamente encuentros de juego y arte en el espacio público como práctica de promoción de salud mental, propiciando vínculos entre instituciones, universidad y comunidad (Bang y Jungberg, 2016).

Las organizaciones que conforman RIOBA se ubican en el barrio de Abasto (ciudad de Buenos Aires) donde se entrecruza un importante centro comercial con una gran población migrante, vulnerada en sus derechos, que es objeto de complejos procesos de desarraigo y constante discriminación (Carman, 2004). Con el objetivo de hacer frente a las diversas problemáticas que emergen en ese entramado social, se crea la Red en 2006. Desde entonces se desarrollan actividades comunitarias, culturales y recreativas de promoción de salud, en un intento por fortalecer el tejido social para la reconstrucción de vínculos solidarios, promover la cultura comunitaria, abordar diversas temáticas que hacen a la salud/salud mental de la población y articular creativamente los emergentes del entramado social del que se participa (Bang, Barile, Guldris y Jungberg, 2018). Las acciones conjuntas de RIOBA se centran en la realización de eventos callejeros con actividades de arte, juego y creatividad, que ocupan participativamente el espacio público. Es en articulación con dicha Red que nace nuestro proyecto de extensión universitaria, como una experiencia participativa de promoción de salud mental desde una perspectiva integral. Si bien ya hemos descrito la experiencia y analizado algunas implicancias en escritos anteriores (Guldris, Bang y Barile, 2017), nos interesa aquí reflexionar sobre algunos otros aspectos que han resonado fuertemente en nosotras/os a partir de nuestra participación en el proyecto.

Derivado de un proceso de investigación previo que concluyó en la tesis doctoral de una de las docentes participantes (Bang, 2014a), cada año el proyecto ha implicado la participación activa en el proceso de organización, realización y evaluación de varios eventos comunitarios y callejeros realizados por RIOBA, proceso que se acompañó de reuniones grupales de reflexión y articulación conceptual entre docentes y estudiantes del proyecto. Durante el año 2015, la participación de los y las estudiantes ${ }^{1}$ se centró en las actividades de la Casona Cultural Humahuaca, abriéndose a otras cuatro instituciones en los años si-

1) Cabe aclarar que algunos participantes, al momento de formar parte del voluntariado, se habían graduado recientemente. De todos modos, los denominamos genéricamente como estudiantes a partir de este rol que se les otorga desde el programa de Voluntariado Universitario. De todas formas, cuestionamos la relación asimétrica que propone la distinción clásica entre docentes y estudiantes en la realización de proyectos universitarios. 
guientes (un centro de salud, un centro de salud mental y dos instituciones con juegotecas barriales). Desde una perspectiva de salud y salud mental integral (Stolkiner y Ardila, 2012; Bang, 2014b) entendemos que el entramado comunitario resultante de este proceso favorece el sostenimiento de vínculos solidarios y el fortalecimiento de la trama de contención comunitaria, posibilitando un abordaje colectivo de diversas problemáticas y temáticas que hacen a la salud y salud mental de la población (Bang, 2016).

En el tránsito por esta experiencia que articula extensión, investigación y docencia, hemos ido construyendo y nos hemos encontrado con algunos atravesamientos que entendemos cobran significación en nuestro contexto de formación universitaria. Nos referimos a una construcción particular del vínculo entre docentes y estudiantes, nuestra participación implicada en las actividades comunitarias y la inclusión de una mirada compleja, integral y reflexiva, con relación al rol profesional y de investigación en lo comunitario. Este proceso nos ha permitido reflexionar sobre la importancia de transitar experiencias comunitarias de forma implicada, crítica y participativa como parte de nuestra formación como profesionales comprometidos con la realidad social, en particular profesionales psicólogos y psicólogas en el contexto de formación universitaria actual. Dicha formación ha estado históricamente caracterizada por una fuerte tensión entre teoría y práctica y entre formación académica y realidad social (Sousa Santos, 1998). Sabemos que tanto el campo de la formación profesional como el de la investigación académica han estado signados por dicha dicotomía. La posibilidad de participar de una experiencia comunitaria desde la vivencia y la experiencia de forma reflexiva nos ha posibilitado la construcción de vínculos significativos no solo entre docentes y estudiantes sino también con referentes institucionales y con la comunidad. A partir de todo ello, nos preguntamos: ¿cómo sistematizar las características significativas de este proceso? ¿Cómo impacta este proceso en la construcción de nuestro rol profesional y docente? ¿Cómo se da la articulación entre formación, extensión e investigación? ¿Qué implicancias tiene la inclusión de un proyecto de estas características en nuestro contexto universitario actual? ¿Qué consecuencias tiene nuestra participación en las prácticas llevadas adelante por las instituciones y organizaciones sociales?

Con estos interrogantes, y a través del análisis de breves viñetas y crónicas vivenciales, nos proponemos abordar los aportes, implicancias y tensiones de la inclusión de prácticas comunitarias vivenciales en la formación universitaria, haciendo hincapié en la tensión entre teoría y práctica y entre formación académica y la realidad social, lo que caracteriza nuestro ámbito académico actual. Esperamos también poder contribuir a la problematización de la relación entre universidad y comunidad analizando las tensiones históricas de nuestra formación entre teoría y práctica, investigación y acción.

Metodológicamente, exponemos diversas viñetas o breves relatos de situaciones que consideramos significativos de la experiencia de Voluntariado Universitario para analizarlos incluyendo reflexiones provenientes de las crónicas grupales y aportes conceptuales que hemos considerado pertinentes. Al tratarse de una experiencia de formación universitaria de la Facultad de Psicología en el campo de la salud mental comunitaria, nos apoyamos en las ricas producciones teóricas del movimiento de salud colectiva (Czeresnia y Freitas, 2006; Merhy, 2006) y la psicología social-comunitaria (Montero, 2003, 2007).

Las autoras y el autor de este escrito somos tres estudiantes de Psicología y participantes del proyecto mencionado en diferentes años (de 2015 a 2017) y una coordinadora docente. 
Nos parece significativo resaltar que esta producción es el resultado del intercambio de vivencias de un mismo proyecto con las particularidades que cada proceso grupal tuvo en cada año. No solo se trata de pensar y repensar nuestros saberes, sino que también se producen colectivamente saberes diferenciales que escapan a las ideas singulares de cada uno. Entendemos que es de esta forma que ha tomado cuerpo la articulación entre extensión e investigación en el proceso realizado.

Primeramente, contextualizaremos el proyecto de extensión universitaria a partir del recorrido histórico que lo ha vuelto posible. Luego presentaremos, a través de crónicas vivenciales y breves viñetas, diferentes escenarios del proyecto de voluntariado universitario, a saber: el escenario callejero, la articulación institucional, el acompañamiento docente, y el recorrido de estudiantes por las instituciones de RIOBA. Por medio de este material, dialogaremos con algunas conceptualizaciones que dan lugar a una articulación y análisis. En tercer lugar, abordaremos la palabra "voluntariado" y analizaremos el sentido que le otorgamos. Finalmente, arribaremos a conclusiones y reflexiones finales sobre el proyecto y sobre el rol de los diferentes actores que forman parte del mismo.

\section{Antecedentes de nuestra propuesta}

Desde la modernidad, la universidad pretende ser el lugar por excelencia de la producción de conocimiento científico, concentrando el prestigio en la investigación pura en desmedro de la investigación aplicada, participativa, y todo otro acercamiento a la realidad social. Autores como Sousa Santos (1998) encuentran allí el origen de la dicotomía entre teoría y práctica y la absoluta prioridad histórica de la primera. En América Latina, además, la universidad ha representado históricamente un modo más de colonización a través de la imposición de la legitimidad de una forma de conocer como válida: la de la ciencia universal y objetiva (Lander, 2000). Según Guelman (2013), este proceso se ha dado en desmedro de cualquier otra mirada catalogada como saber ilegítimo, bárbaro y superfluo.

Estas formas de ejercicio del poder-saber han tenido también fuertes cuestionamientos desde sectores de la propia institución universitaria desde hace décadas. Es así que, a partir de los años 60 , se realiza un fuerte llamado a la práctica, cuya vertiente sociopolítica se tradujo en la crítica del aislamiento de la universidad, de la torre de marfil insensible a los problemas reales del mundo contemporáneo (Sousa Santos, 1998). Se ha impulsado así la concepción de responsabilidad social de la universidad (Buarque, 1986), entendiendo que la política de la universidad debe combinar el máximo de calidad académica con el máximo de compromiso social. En este contexto nacen las políticas de extensión, con la consideración de que el conocimiento científico, tecnológico y artístico generados en la universidad no son únicos. Se reconocen otras formas de conocimiento surgidas de la manera de pensar y actuar de los innumerables segmentos de la sociedad a lo largo de generaciones que, por no estar caracterizadas como científicas, están desprovistas de legitimidad institucional e invisibilizadas (Sousa Santos, 1998). A pesar de ello, en la práctica, las actividades de extensión muchas veces han procurado "extender" la universidad sin transformarla, traduciéndose en aplicaciones técnicas y no en aplicaciones edificantes de lo nuevo, producto de un encuentro. 
En consecuencia, entendemos que aún es un desafío central de la formación universitaria el afrontar esta falta de articulación histórica entre teoría y práctica. Dicha característica, propia de gran parte de la enseñanza universitaria, produce un gran aislamiento del conocimiento con respecto al contexto histórico-social donde acontecen los problemas. En este sentido, Ivanna Petz (2015) propone pensar la universidad en relación con, entendiendo la importancia de considerarla actor social interviniente, como parte del territorio constituido y entrelazado por otros actores. Entendemos que, para esta transformación, las relaciones de conocimiento en la universidad deben comenzar a incorporar experiencias comprometidas con lo social, que den importancia a lo vincular y afectivo en la construcción conjunta de una mirada implicada sobre la realidad.

Es desde esta perspectiva que nuestro proyecto de Voluntariado Universitario surge y se articula con un proceso de investigación previo incluido en un proyecto de investigación UBACy $^{2}$ de la misma cátedra en la que lo sostenemos hoy. A partir de 2009 se desarrolló una investigación participativa que tuvo como sujeto y objeto de investigación a las organizaciones de la red RIOBA, y que concluyó en la tesis doctoral de la docente e investigadora Claudia Bang (2014a). Dicha producción se constituyó en un fuerte antecedente a la presente propuesta y dialoga permanentemente con ella. Los vínculos producidos entre investigadores y comunidad en ese proceso de investigación participativa abonaron la posibilidad de planificar un proyecto de extensión como un verdadero proceso colectivo. La sistematización de todo ello pretende hoy aportar también al fortalecimiento de esa articulación entre extensión universitaria, formación profesional y un proceso de investigación comprometidos con la realidad social.

\section{El escenario callejero}

Un primer problema que nos inquieta es sobre el modo en que adquirimos los conocimientos en la universidad y cómo este modo construye nuestro rol, nuestro posicionamiento futuro de trabajo como profesionales de la salud y también como docentes e investigadores. La manera en que aprendemos no está solo en nuestra relación con los libros sino también con los y las docentes, con nuestros compañeros y compañeras y con la realidad social; asimismo, con la indagación que podamos hacer de nosotros mismos sobre aquello que conocemos.

Hoy en día, muchas materias que cursamos como estudiantes en la universidad se basan en un aprendizaje por medio de la repetición de contenidos más que en un aprendizaje por la experiencia. Así, la posibilidad de escribir sobre los eventos, las reuniones y los distintos espacios donde se desarrolla el Voluntariado Universitario nos acerca a lo vivido, a nuestro estar presente de forma disponible y abierta a lo que sucede. En este sentido, tomamos de Larrosa (2003) la idea de que como sujetos de la experiencia, nos encontramos expuestos, con todo lo que eso supone de vulnerabilidad y riesgo. Es por eso que hemos decidido partir de crónicas y viñetas de las diversas experiencias que forman parte del Voluntariado Universitario para reflexionar sobre los interrogantes que nos atañen. A partir de este ma-

2) Se trata del proyecto UBACyT "Articulaciones entre salud mental y atención primaria de la salud desde una perspectiva de derechos - Argentina - 2004 y 2014" presentado bajo la dirección de la Dra. Alicia Stolkiner. Instituto de Investigaciones, Facultad de Psicología UBA. 
terial vivencial, podremos realizar algunas articulaciones que nos permitan conceptualizar nuestras prácticas.

Comenzaremos con algunos fragmentos de crónicas realizadas por estudiantes en los años 2015 y 2016 sobre un evento callejero organizado para festejar el cumpleaños colectivo de distintas organizaciones:

Estoy llegando a la Casona Cultural Humahuaca para celebrar, en un Festival Callejero, el cumpleaños de ese centro cultural, junto con el cumpleaños del CUJUCA —Cumbre de Juegos Callejeros-y la FLIA —Feria del Libro Independiente y Autogestiva.

Me voy acercando y veo que la calle aún está vacía, pero con varias manos trabajando, pintando distintos juegos en la calle. En la cuadra que le sigue, la feria se está montando. Algo que me llama mucho la atención es que las mesas están afuera también ¡Un bar montado en el medio de la calle! A lo lejos veo a una compañera del voluntariado pintando la calle. Me encuentro con otra compañera, se la ve muy contenta. Juntas vemos cómo va quedando el puesto de la feria de La Vereda - Institución que forma parte de la Red RIOBA y del proyecto de voluntariado- con todos los tejidos expuestos, está lindo, colorido. Vamos a chusmear la FLIA. Pienso que me quiero llevar todo. Es temprano y ya hay mucha gente, mucho movimiento. Seguimos recorriendo las otras calles. No me quiero perder nada.

Nos cruzamos a la primera compañera que vi de lejos, que tiene las manos cansadas de tanto pintar. Nos muestra muy contenta el cartel que hicieron del Hospital Ameghino -Institución que forma parte de la Red RIOBA-, lleno de fotos del laboratorio de objetos - taller del $\mathrm{H}$. Ameghino orientado a niños que participa del voluntariado.

Me detengo a ver la calle de nuevo y la encuentro completamente distinta: los juegos ya están montados. En la calle propiamente dicha se ven muchas rayuelas de todos los colores y formas; un circuito; la pista para la carrera de embolsados; la pista de autitos; el tatetí humano; entre otros. En las veredas se encuentran montados más juegos y talleres: el cuentacuentos; el tumbalata; un taller de arte; entre otros. La calle ya es una fiesta.

Traen los paracaídas de colores. En el primer juego somos chicos y grandes, todos se divierten. Se arma una ronda con todos en el cruce de calles, somos un montón.

Cantamos, jugamos, corremos. Hacemos el mítico "Cujuca, que?!": una canción que da la bienvenida a los juegos.

Con otra compañera vamos hacia el juego de la soga, que parece ser la más convocada. Salto, pero pierdo siempre en "Cielo". Pensé que era mejor saltando, esto me da gracia, me divierto igual. Me había olvidado de todas las cancioncitas que se podían cantar saltando. Las voy recordando.

Me emociona conocer las reglas de los juegos, seguirlas, jugar. También miro cómo juegan. Me sorprende la soltura de los adultos jugando, nadie parece tímido. Hay mucha gente. muchas voces, muchas sonrisas. Me dirijo a la carrera de embolsados. Entre coordinadora y participante, me quedo un buen rato disfrutando. Jugamos a las carreritas en varias de sus versiones: con zancos, embolsados, carretilla y demás.

Dan por terminado el CUJUCA, se organiza la merienda en el sol pintado en el cruce de calles. Ubican unas mesas. Me siento en el piso con los demás compañeros del voluntariado. De repente están todos con un vaso de chocolatada caliente y una galletita en la mano. Alguna de las chicas dice algo muy cierto: "qué loco pensar que estamos todos sentados en la 
calle, por donde suelen circular los autos”. Los más chicos pareciera que no piensan en eso, ellos vinieron a jugar y jugar se puede en cualquier parte. Escucho que una madre le dice a su hijo que si quiere chocolatada que le pida a los grandes. Él le dice que no hace falta pedir, que ya le van a dar. Está confiado en que él es parte de lo que está pasando y que no se van a olvidar ni de él ni de su chocolatada. A nosotras también nos convidan. Por momentos somos niñas y por otros, adultas.

Creemos que la experiencia en el hacer, en el contacto con la realidad, con otros reales distintos a nosotros y nosotras, nos compromete y nos conmociona. Este aprendizaje a través de la vivencia nos enriquece en tanto nos hace presentes como personas, estudiantes y futuros profesionales. La experiencia nos acerca a un segundo problema, que se corresponde con la definición del rol del voluntariado: al no ser un rol definido, la experiencia en su incertidumbre habilita cuestionarnos sobre nuestro hacer, sobre el modo en que observamos, sobre cómo nos presentamos y esta imprecisión nos mantiene activos y curiosos.

A partir de las crónicas vivenciales en las cuales hemos plasmado algunos de los momentos que hemos transitado durante la experiencia del voluntariado, reconocemos estos espacios donde aparecen las dudas y la observación constante. Mientras el aprendizaje sea por medio de la experiencia podremos producir saberes con sentidos significativos y no solo cambios conceptuales, sino tener la posibilidad de comprender genuinamente las problemáticas para reflexionar y elaborar propuestas alternativas.

Larrosa (2003) define a la experiencia como "aquello que nos pasa", pero caracteriza al mundo en que vivimos como pobre de experiencias, ya sea por exceso de información, de opinión o por falta de tiempo, entre otras cosas. La experiencia no solo nos pasa sino que también exige ser registrada. La posibilidad de que algo nos acontezca hoy en día, dice Larrosa, requiere detenernos, pararnos a pensar, a escuchar, a mirar, a darnos tiempo y espacio para observar con todos nuestros sentidos, algo que se nos dificulta en los tiempos que corren. Para experimentar deberíamos habitar un sujeto de la experiencia y no de la información, "una superficie de sensibilidad en la que lo que pasa afecta de algún modo (...) inscribe algunas marcas." (2003:175). Así, en clave vivencial, el sujeto da paso a lo rico de la experiencia.

En consonancia, Agamben nos dice que la experiencia es "incompatible con la certeza" (2011:14), y agrega que si una experiencia se convierte en "calculable y cierta pierde inmediatamente su autoridad. No se puede formular una máxima ni contar una historia allí donde rige una ley científica" (Agamben:14). Es decir, la experiencia nos invita a salir de la mirada cierta e imperativa de la concepción positivista. El registro de la experiencia y su riqueza nos permite dudar, dejarnos llevar por la incerteza. De esta forma, dejamos de ser los sujetos impasibles, que no muestran emoción, o reacción frente a lo que sucede. Las experiencias no nos permitirían fijeza, certeza. La captura de la experiencia se convertiría en experimento, lo que Agamben entiende como la certificación científica de la experiencia. Si hacemos una captura de la experiencia perderíamos lo vivo de su material, que implica los atravesamientos subjetivos de quienes las vivimos.

Entonces bien, adentrándonos en la viñeta que nos compete, aparece incesantemente un sentimiento de sorpresa. Ella representa la inexistencia de un quehacer delimitado y planificado en su exactitud, de antemano. Claro que, a modo de cartografía, hay trazos dados: ese festival fue organizado por la comunidad en su conjunto, planeando actividades, horarios, la 
merienda, ultimando detalles en un trabajo colectivo. Se trata de trazos que de ningún modo se presentan cerrados, sino que, al contrario, marcan el curso de lo que luego se dibuja y desdibuja en el acontecer espontáneo. La cartografía, explican Franco y Merhy, "es un dibujo que acompaña y se hace al mismo tiempo que los movimientos de transformación del paisaje" (2011:14). Así, tanto la sorpresa de "un bar montado en medio de la calle", como la de la apropiación del espacio para trazar los juegos, anuncian la deconstrucción de un territorio existencial, es decir, de "aquello que cada uno tiene dentro, y que define su forma de significar e interactuar con el mundo" para, en el proceso de participar activa y espontáneamente de la puesta en marcha del festival, trazar nuevas líneas desde el afecto, que transforman al sujeto, al mismo tiempo que éste influye sobre la realidad (Franco, Merhy:14). Duschatzky, Farrán y Aguirre (2010) señalan la importancia de la participación fundada en el afecto y la proximidad, que acortan las distancias entre los miembros y hacen factible la recreación de un lazo de confianza y proximidad que, construyéndose en el momento mismo en el que son vivenciadas, escapan a todo protocolo.

Sin embargo, como decíamos en un escrito anterior (El Entre, 2015), asumir el desafío de deconstruir nuestros preconceptos, no implica que sea una experiencia donde todo queda librado al azar y la sorpresa. Se trata, en cambio, de una experiencia que se afirma en el desafío de acompañar procesos que ya son parte de la comunidad, asumiendo una posición más horizontalizada y respetuosa de las singularidades, sin perder de vista todo lo que cada una y cada uno puede aportar en ese proceso.

\section{La articulación institucional y el acompañamiento docente}

Entendemos que una de las características distintivas de nuestro proyecto es que su planificación se da en plena articulación con instituciones y comunidad. A continuación, presentamos un breve relato acerca de una reunión de planificación entre referentes comunitarios y una de las docentes del proyecto:

Me encontré con dos referentes institucionales a mediados del año 2015 para evaluar el proceso de voluntariado y comenzar a planificar el proceso del año siguiente. Guille y Mariela son los dos referentes de la Casona Cultural Humahuaca, institución desde donde se desarrolló el primer proyecto. Mariela además, participa de la coordinación del proyecto y de las reuniones de reflexión. Una de las cuestiones centrales era saber qué aportó la participación del proyecto a la vida de la Casona Cultural:

Guille cometa que "el grupo estuvo muy enganchado y le dio mucha fuerza al proceso del área comunitaria en su conjunto. Se hicieron entrevistas para una radio comunitaria y se articularon muchos procesos. La participación desde el voluntariado de estos estudiantes o jóvenes profesionales a las actividades comunitarias en el Abasto facilitó la conformación grupal y le dio más cuerpo a las actividades." Con relación al proceso reflexivo conjunto afirma: "También nos ayuda a pensar nuestras actividades, las que venimos haciendo hace mucho tiempo como promotoras de salud y salud mental, eso era algo que no habíamos pensado realmente antes... que venga gente de la facu de psicología de una materia de Salud significa que nosotros tenemos algo que ver con eso, con procesos de salud en la comunidad. A la Casona le da vitalidad y la circulación de estudiantes universi- 
tarios genera otra cosa... desde siempre quisimos articular con universidades, también da otra impronta ante el barrio...".

Mariela dice que para ella fue un proceso con mucho desafío docente: "Yo imaginaba que en las reuniones íbamos a hablar de cosas conceptuales y teóricas de la facultad, pero no fue así, fui sintiendo que podía aportar mucho al proceso desde mi lugar y que se conformó un muy buen proceso grupal y vincular". Las reuniones de formación y acompañamiento se articulan a un proceso muy vívido, donde un espacio se nutre del siguiente, eso ha facilitado que se traslade algo de la "amorosidad" que el grupo reconoce como característica de los eventos a nuestro proceso y reuniones.

Entre mates y charlas, proponemos dar un paso más y para el año siguiente abrir la participación a otras instituciones de RIOBA. Guille dice que "RIOBA está esperando la participación del voluntariado, siempre lo dicen desde el Centro de Salud o la juegoteca". Yo recordé que uno de los motores del armado del proyecto fue que, en varias oportunidades en reuniones de red y desde referentes de instituciones que estaban vinculados a la Universidad trajeron la inquietud de poder articular con algún proyecto de extensión para que estudiantes de la facultad puedan sumarse al armado de los eventos y todo lo relacionado a ello, a fomentar la participación del barrio, la difusión de los eventos, la posibilidad de tener recursos económicos, etcétera.

Así fue cómo al año siguiente participamos en actividades en otras instituciones de RIOBA, como lo relata el siguiente fragmento de crónica de una reunión de reflexión del equipo, donde estuvimos presentes docentes y estudiantes:

Esta semana nos reunimos las coordinadoras del voluntariado y las estudiantes que participamos para contar por primera vez nuestras experiencias en los distintos espacios de las instituciones a las que estamos asistiendo.

Cuando llega mi turno comienzo a contar de la experiencia que había tenido en el espacio de La Vereda con Julieta, otra compañera estudiante. Cuento que antes de empezar en el espacio del taller tuvimos una reunión con Lucrecia, nuestra referente institucional y trabajadora social de La Vereda. En esa reunión, donde nos conocimos, Lucrecia nos cuenta un poco de la historia del lugar y de la creación de los diferentes talleres. También nos muestra todo el espacio de La Vereda y llegamos a conocer a algunas de las personas que trabajan allí. Luego cuento un poco de lo que fue el taller de la juegoteca a donde van chicos de 3 a 8 años, donde con Julieta fuimos observadoras (así lo pactamos con Lucrecia y las demás coordinadoras del taller). Vimos cómo funciona todo el dispositivo, algunas de las reglas que mantienen las coordinadoras y al final, charlamos un poco con ellas contándoles nuestras primeras impresiones y lo que habíamos sentido. Julieta agrega a mi relato que ambas nos habiamos sentido muy bien, muy cuidadas y que por sobre todas las cosas, nos había parecido un poco extraño permanecer solo como observadoras en el taller. Así fue como otras compañeras fueron contando sus primeras participaciones en los diferentes espacios institucionales.

Una particularidad de este proyecto es que fue ideado, planificado y gestionado desde un comienzo por referentes comunitarios y docentes universitarios, quienes veníamos trabajando juntos desde hacía años. En principio el proyecto respondió a una necesidad 
económica y de recursos humanos para el sostenimiento de las actividades comunitarias en el barrio, pero fue mucho más lo que aportó este encuentro a todos los actores intervinientes. Se establecieron relaciones significativas desde las prácticas y el hacer cotidiano, las que se extendieron simbólicamente desde la comunidad al mundo universitario. Ese mundo cuyo imaginario está signado en el barrio por la distancia y la indiferencia ahora estaba allí, jugando con ellos en la calle, participando activamente de los eventos comunitarios. Esto fue posible gracias a que el proceso lo pudimos vivenciar, no solo poniendo palabras a una práctica, sino dejándonos atravesar por ella, movilizándonos desde las sensaciones y lo afectivo.

Otro aporte significativo está relacionado con haber contribuido a la visibilización de la relación de estas actividades con una perspectiva de salud/salud mental integral. Como parte del proceso de acción y reflexión, comenzamos a nombrar lo que allí en la calle venía ocurriendo hacía años como una experiencia de promoción de salud mental comunitaria, como parte del campo de prácticas sociales en salud. Esto impactó fuertemente en los referentes institucionales y comunitarios, quienes comienzan a reconocer allí los elementos de una práctica de salud integral. Actualmente hay una apropiación creciente de estas ideas (muchas de las que se han elaborado en conjunto) por parte de la comunidad, legitimadas en parte por la presencia de la universidad en el territorio. En este sentido, la experiencia se enfoca también en producir conocimiento colectivamente. No solo se trata de pensar y repensar nuestros saberes, sino que también se producen saberes diferenciales que escapan a las ideas singulares de cada uno y cada una.

Consideramos que el entramado resultante de todo este proceso ha sido facilitado y potenciado fuertemente por las relaciones de enseñanza-aprendizaje que hemos construido y sostenido al interior del proyecto. Desde el rol docente hemos acompañado el tránsito de los estudiantes por los diferentes espacios comunitarios, aportando herramientas para la reflexión de una práctica situada, abriendo y sosteniendo espacios de intercambio que se han construido colectivamente. Ir trabajando sobre los emergentes de la práctica, así como desde los intereses e inquietudes de los estudiantes nos ha permitido cuestionar en acto las relaciones de poder docente-alumno hegemónicas en el ámbito universitario. Guelman (2013) afirma que la forma de la enseñanza que asumen las prácticas de extensión universitaria están alejadas de lo escolarizado: plantean otras maneras de construir aprendizaje, otros formatos que el del aula, otros modos de entender la formación y de evaluar otras formas de autonomía de los estudiantes. Así, los espacios de reflexión conjunta han estado cargados de análisis y reflexiones acerca de los múltiples atravesamientos y las tensiones de las prácticas comunitarias, las características de los procesos participativos, la dificultad de definir y delimitar a la comunidad y al territorio, la posibilidad de pensar características de la intervención y de los diversos campos profesionales, etc. Para ello partimos de la elaboración de crónicas vivenciales, reconociéndonos también como sujetos de participación; es desde ese lugar que nos acercamos a la experiencia, a poder reflexionar sobre ella y articularla con una rica trama conceptual. Esto nos ha permitido desdibujar, por momentos, la distancia clásica —el abismo- entre teoría y práctica, y lograr una articulación dialéctica entre los elementos en juego.

Uno de los elementos más significativos ha sido la posibilidad de construir en este proceso un enfoque epistemológico compartido: pensar la realidad de forma múltiple y compleja, permitiéndonos dibujar algunos trazos sobre las implicancias de nuestra práctica allí 
situada. El entramado de muy diversos saberes presentes en las prácticas de RIOBA nos ha hecho nutrirnos y construir miradas compartidas de forma interdisciplinaria, donde dialogan saberes provenientes de la psicología, la medicina, la recreología, la educación popular, el psicodrama comunitario, la comunicación social, saberes populares y de la historia barrial, saberes artísticos y tantos otros. La co-coordinación del proyecto con una referente comunitaria, la participación de otros referentes en los espacios de reflexión conjunta, así como el encuentro y diálogo permanente con muchos otros, han sido las vías de posibilidad de esta construcción de miradas compartidas. Se trata también de una construcción interdisciplinaria de nuestras propias prácticas, resultante de un ejercicio permanente de apertura y flexibilidad. Entendemos que el proceso tendiente a la horizontalización de los vínculos docente-estudiantes y universidad-comunidad antes expuesto nos ha facilitado este movimiento de construcción conjunta en intersaberes.

\section{En las instituciones de RIOBA}

El "Taller de laboratorio creativo" del Centro de Salud Mental $N^{\circ} 3$ Ameghino se trata de un dispositivo en donde todos los encuentros son distintos y la incertidumbre la misma: "¿qué harán hoy los chicos en el taller?", me preguntaba camino al hospital. Puede decirse que lo único que se mantiene siempre es la consigna del desarrollo libre de la actividad de creación y el espacio en donde se realiza. Es que justamente de eso se trata: no hay demasiadas reglas ni explicaciones de cómo hacer, la libertad de la actividad es lo primordial. Es por eso que al ingresar a uno de los salones del hospital, los chicos de entre 6 a 12 años tienen a su disposición distintos materiales (elementos descartables que podrán ser reciclados, telas, marcadores, pinturas, etc.) con los que podrán crear, jugar, o explorar. Al ingresar, uno de los niños se dirige a la ventana para ver qué puede utilizar. De pronto observa que detrás del armario hay un maniquí. “¿Me ayudas?”, me dice, tratando de agarrar del brazo al maniquí. Una vez de frente a éste, comienza a buscar telas en una caja que trae la psicopedagoga. Allí encuentra distintos retazos, insumos necesarios para vestir a su modelo. Mientras el niño realiza muy concentrado esta actividad, uno de sus compañeros junto con una de las psicólogas, juegan a la pelota-paleta con dos pedazos de cartón y una pelota hecha de papel y cinta (minutos antes) en un extremo del salón. Por otro lado, otro de los chicos junta tubos de cartón y los apila: "¿qué es?" - le pregunto-; "una espada", me responde casi sin levantar la mirada de sus elementos. Trato de ver en qué colaborar, en qué ayudar. En ese entre hacer y no hacer, o solo alcanzar la pelota del partido no sé bien cómo pararme, ¿qué debería hacer? ¿Cuál es mi rol? ¿Esto tiene que ver con la psicología? Son algunas de las preguntas que se repiten en cada encuentro. Entre risas, anécdotas y participación activa, transcurre la hora del taller.

A partir de esta viñeta, podemos entender que lo que ocurre en el taller es lo que Franco y Merhy llaman un trabajo vivo en acto, definiéndolo como "proceso agenciado por sujetos, que trae en sí el atributo de la libertad, de la creación, de la inventiva" (2011:13). Es allí donde, frente a un quehacer específico sustentado en un imaginario de lo que debemos realizar como futuros psicólogos, surge entre quienes transitamos estos espacios un razonamiento acerca de la actividad que creemos pertinente en el momento, no pensada a 
priori. Tal como señalan estos autores, partiendo de una situación relacional en donde lo que sucede es siempre en acto, pueden encontrarse allí producciones del cuidado en salud, donde se desplegará cierta afectividad propia de la relación. A su vez, aun guiados por una misma consigna - desarrollo libre de la actividad de creación y el espacio en donde se realiza - podría hablarse de una singularidad en el proceso de trabajo, donde cada actor realiza una práctica de cuidado apelando también a su propia creatividad. En consonancia, a la hora de ubicarnos en un rol, se puede decir que hay un proceso de subjetivación, una producción de sí en donde quienes estamos implicados en la actividad nos vemos en la necesidad de reconocernos afectados por el objeto de trabajo, el cual nos modifica. Hay una cartografía, en tanto que no habrá una sola forma de hacer, sino una entre muchas opciones, de acuerdo a diversos factores que influyen en la situación, entre ellos el vínculo, el afecto, las singularidades producto de una construcción entre cuidador y cuidado, y a la inversa. Habrá una verdadera desterriorialización (Franco y Merhy, 2011), deconstrucción de lo esperable, donde se deberá romper con el lugar de origen, en este caso la universidad como constructora de limitados territorios de acción. En la práctica, sucede que es ella misma la que nos guía, o nos enseña cómo trabajar, nos muestra una forma de hacer como profesionales que va más allá de lo dictado por cierta teoría. Lo diferente del día a día de las personas con las cuales tratamos hace que nuestros preconceptos muchas veces no se adecuen con eso que se nos presenta, con lo que interactuamos. Podría pensarse que ese ponernos a prueba respecto de los saberes aprendidos nos lleva a indagar otros espacios, otros conocimientos, pudiendo delimitar y marcar una (o varias) formas de trabajo que consideramos pertinente. Esto servirá para ir en busca de nuevas identidades en nuestras prácticas.

Mientras que en muchos espacios de la facultad nos enseñan que lo clínico sucede solo en el espacio de consultorio e implica definir categorías o patologías, en la realidad lo clínico es mucho más amplio. Tal como plantean Stolkiner y Ardila (2012), se trata de tener en cuenta la dimensión histórica y social del sujeto, trascendiendo la ontología de la enfermedad, pudiendo incorporar procesos de trabajo de diferentes áreas. Entonces, estas experiencias permiten romper con el imaginario: no es sólo hacer en los talleres sin más, sino que son abordajes que tienen su base teórica y su legitimidad como cualquier otro espacio terapéutico. Asimismo, este abordaje permite rearticular el abismo entre teoría y práctica que se da en la formación académica tradicional, ya que la práctica en la institución admite una articulación concreta entre la experiencia y su base conceptual.

Es con esta idea de comprender a la salud mental en sentido amplio que nace el Taller de Laboratorio Creativo. Quienes lo idearon forman parte del equipo de profesionales del área infanto-juvenil del hospital, teniendo en cuenta algunas de las problemáticas de sus pacientes, pero invitando a participar a cualquier otro niño que no necesariamente asista al hospital. Sucede que no todos los niños tienen ese espacio físico para jugar en su casa, ni tampoco un grupo de pares para desarrollarlos. Es así como este dispositivo se inicia desde la idea de que los cuidados en salud mental no solo se llevan a cabo dentro de un consultorio, en una terapia individual. Compartir con otros, jugar, interactuar y tener ese momento a la semana para desplegar las potencialidades de la imaginación también es salud y da lugar a la creatividad entendida como "un proceso dinámico de la subjetividad humana, que articula la dimensión individual y social en la intersubjetividad" (Bang, 2012:17). A su vez, y 
como otro motivo de legitimación, en el taller se fabrican las piezas y los juguetes que luego serán quemados en la fiesta de la Quema del Muñeco, evento organizado por RIOBA, al cual también asisten los participantes del taller junto con sus familias. Se trata de un momento de unión, encuentro y visibilización de la práctica. Porque en el centro de salud no solo se va a "la psicopedagoga", también se va a crear y jugar. En este sentido, el marco normativo vigente reivindica este tipo de prácticas en su aporte a la salud. Por ejemplo, la Organización Panamericana de la Salud (2009) expresa que el arte es una "poderosa herramienta promotora y reparadora de la salud" que promueve escenarios más felices para la vida de las personas. La Ley Nacional de Salud Mental 26.657 (2010) exige y legitima la implementación de prácticas de Atención Primaria de la Salud con base comunitaria que fortalezcan el entrelazamiento social. Conceptualmente, estas prácticas son entendidas desde de la Medicina Social/Salud Colectiva Latinoamericana como promotoras de salud y salud mental (Czeresnia y Freitas, 2006), fruto de una combinación de estrategias establecidas junto con cada población (Bang, 2016). En consecuencia, podemos entender al taller como una estrategia válida y legítima de promoción, pensada y desarrollada a partir de las particularidades de la población a la cual está destinada.

Ulloa (1996) retoma el valor de la ternura como potencia y resistencia frente a lo mortificante de la sociedad. Es en ella que se vuelve posible la construcción de un vínculo enmarcado en lo que el autor conceptualiza como buen trato, diferenciándose del tratamiento. Mientras este último supone la patologización de los padecimientos y la búsqueda de su correspondiente cura, dando lugar a una estandarización que anula al sujeto, y desliza fácilmente el tratamiento hacia el maltrato, el buen trato implica la valorización de las singularidades en sí mismas. Este se funda, como anticipábamos, en la ternura. De este modo, podemos pensar al taller (como el resto de dispositivos que se articulan en los eventos callejeros de la Red) como un dispositivo que se afirma en el encuentro de los estudiantes con la ternura y forja a partir de ella un vínculo que no tiene una teoría explicitada de antemano, que no invita a pensar el juego como una mera terapéutica con relación a una patología, sino que instaura en el juego una herramienta que habilita el encuentro y la construcción de vínculos mediados por la ternura. Al mismo tiempo, la participación en el juego involucrando el cuerpo y los afectos, como humano en su totalidad, permiten el pasaje de estudiante pasivo, observador de teorías, a un estudiante activo, constructor de la propia realidad que él mismo habita.

\section{Revisión del sentido del término "voluntariado"}

En los diferentes encuentros grupales de análisis hemos coincidido en la percepción de no sentirnos respaldados en la terminología "voluntario" tal cual se presenta en el imaginario social. Así, podría pensarse el rol del voluntario y la voluntaria como quien aporta algo de lo que le sobra (sea tiempo, o bien cosas materiales de las cuales puede prescindir), y a la comunidad como quien recibe, lo que generaría un compromiso que, en palabras de Maritza Montero, se vuelve una "zona de penumbra ubicada entre la beneficencia social y el narcisismo socializado, (...) como una disposición benevolente de los agentes externos, deseable y correcta, sobre todo políticamente correcta." (2007:241).

Por otro lado, podríamos decir que estos proyectos de extensión constituyen otros territorios a explorar, existenciales y situacionales distintos a los construidos en la formación 
obligatoria de grado. En este sentido, durante las reuniones del voluntariado 2017, surgió entre algunos de los participantes la idea de ubicar al proyecto en analogía con una práctica profesional, como materia de la carrera universitaria. Si bien la organización del proceso realizado podría tener similitud con dichas prácticas, es preciso reconocerlo como otro lugar aun estando dentro de la facultad. Pierde su similitud en tanto que se ubica como un proyecto de extensión de carácter no obligatorio, en el que los participantes no debemos obtener cierta calificación ni estamos siendo evaluados, donde los roles de educador y educando no son los estereotipados y es factible encontrar una horizontalidad. No obstante, el punto en común lo encontramos en el hecho de que, no obstante tener estas características, no deja de ser un espacio de formación en el cual se aprende a hacer en situación como respuesta a una problemática de la comunidad.

Si revisamos entonces el sentido que le otorgamos al término voluntariado y al proyecto, nos pensamos a partir de un compromiso sostenido en la premisa del respeto por el otro y de un derecho al acceso a la salud mental ampliamente entendido. Concebimos al otro en su integridad, como poseedor de historias, de saberes, de percepciones del mundo. Cada uno tiene sus diferentes saberes, y es en la multiplicidad que emerge la construcción colectiva como potenciadora de la transformación social y de lo saludable.

La tarea de quienes participamos del proyecto se extiende. Implica acercarse al mundo con la curiosidad latente que habilite el acontecer espontáneo. Existen, claro, reflexiones teóricas con las cuales dialoga, pero la vivencia escapa a toda anticipación. En efecto, es la incapacidad de anticipar la que permite el encuentro horizontal con los miembros de la comunidad. Aparece entonces un vínculo de intercambio que incluso excede a la bidireccionalidad: se transforma en "flujos de intensidades continuas entre los sujetos que actúan en la construcción de la realidad social" (Franco, Merhy, 2011:10) Allí donde laten las preguntas y la predisposición a la construcción colectiva de respuestas es, pues, donde nace nuestro trabajo.

\section{Reflexiones finales}

En este trabajo hemos ensayado algunas respuestas a las preguntas que nos planteamos en la introducción, resaltando lo fuertemente significativo que este proceso ha sido para la construcción de nuestro rol profesional y docente.

A partir de lo relatado es que, junto con otras tantas prácticas comunitarias, de investigación participativa y de extensión universitaria, asumimos el desafío de contribuir a abrir caminos en el vínculo universidad-sociedad. Junto con Guelman (2015), entendemos que la universidad puede transformarse también por medio del conocimiento que construya en el vínculo con el afuera y sus sujetos, tratándose del reconocimiento de la bidireccionalidad.

Reflexionamos también acerca de la forma de surgimiento del proyecto de extensión. La red RIOBA y los eventos callejeros existían antes de que la Universidad formara parte de ellos, antes de ser objeto de investigación o práctica de extensión, y desde ese reconocimiento es que hemos construido conjuntamente esta experiencia. Es decir, el proyecto de voluntariado se ubica fuera de la lógica del pensamiento clásico que postula un camino desde la academia hacia la comunidad no académica. Si bien surge de la necesidad de encontrar un apoyo para la continuidad de los eventos, esto no supone la necesidad de un saber 
universitario-académico previo sobre esas prácticas. Se podría plantear a nuestro proyecto de voluntariado como un dispositivo contracultural, donde se modifican las relaciones clásicas de poder (práctica surgida sobre la base de la teoría) para dar paso a una legitimidad de saberes conjuntos que posiblemente no sean reflejados en la mayoría de las aulas.

Acordamos con Sousa Santos (1998) que la práctica social que produce y se sirve del saber científico es una entre otras. La universidad debe participar en la definición de las potencialidades y de los límites de esta práctica en el contexto de otras prácticas sociales donde se manejan otras formas de conocimiento: campesino, técnico, popular, cotidiano, artístico, religioso, literario, etc. Sentimos un gran desafío en poder acercar estos mundos: el de la universidad y las ideas con el de las prácticas comunitarias y concretas. Asumimos el desafío porque nos reconocemos también en ese entre.

Reafirmamos el valor de nuestra participación como docentes y estudiantes activos, críticos, y reflexivos con relación a la realidad en la que vivimos. Valoramos la posibilidad de empoderarnos como sujetos y como futuros psicólogos para, en un trabajo conjunto con la comunidad, hacer frente a los avatares de la realidad social. Este proceso nos permite asumir conjuntamente la responsabilidad que implica pensarse como partícipes de una transformación social que no solo nos atraviesa en el accionar concreto, sino que se extiende al cúmulo de decisiones que deben ser tomadas a diario. El aprendizaje sobre la construcción de una perspectiva crítica en cuanto a la realidad y el actuar propio, se nos presenta como andamiaje indispensable para el trabajo como profesionales de la salud.

Concluimos estas reflexiones con las palabras que ya en 2015 consideramos como claves de esta experiencia, en tanto práctica de promoción de salud mental comunitaria: compromiso, cooperación, perseverancia, amorosidad, iniciativa, firmeza, determinación, intervención activa, constancia, grupalidad, sensibilidad, creatividad y comunicación (El Entre, 2015).

\section{Referencias bibliográficas}

Agamben, G. (2011). Infancia e Historia. Buenos Aires: Adriana Hidalgo.

Bang, C. (2012). El juego en el espacio público y la participación comunitaria: una experiencia de promoción de salud mental en la comunidad. Lúdicamente, 2, 1-20. CAICYT-CONICET.

(2014a). Promoción de salud mental y prácticas participativas de arte, creatividad y juego: La red RIO-

BA. Tesis doctoral no publicada. Facultad de Psicología, UBA:

(2014b). Estrategias comunitarias en promoción de salud mental: Construyendo una trama conceptual para el abordaje de problemáticas psicosociales complejas. Psicoperspectivas: Individuo y sociedad, 13(2), 109-120. Valparaíso, Chile.

(2016). Creatividad y salud mental comunitaria. Tejiendo redes desde la participación y la creación colectiva. Buenos Aires: Lugar Editorial.

Bang, C.; Jungberg, M. (2016). Proyecto de Voluntariado Universitario "Arte, juego y salud mental. Documento en línea. Extraído el 28/03/2018 de http://www.psi.uba.ar/academica/carrerasdegrado/psicologia/sitios_catedras/obligatorias/066_salud2/index.php?var=actividades/extension.php

Bang, C.; Barile, C.; Guldris, M.; Jungberg, M. (2018). Arte, juego y salud mental: Una propuesta de articulación entre universidad, instituciones y comunidad. Redes de Extensión, Número 4: 27-42. Facultad de Filosofía y Letras, UBA.

Buarque, C. (1986) Uma Ideia de Universidade. Brasilia: Editora Universidad de Brasilia. 
Carman, M. (2004). La ciudad visible y la ciudad invisible: El surgimiento de las casas tomadas en Buenos Aires. Población y Sociedad, (10/11), 71-108.

Czeresnia, D.; Freitas, C. (2006). Promoción de la salud: conceptos, reflexiones. Buenos Aires: Lugar Editorial. Duschatzky, S.; Farrán,G.; Aguirre, E. (2010). Escuelas en escena. Una experiencia de pensamientos colectivo. Buenos Aires: Paidós.

El Entre (2015). Presentación de la experiencia de trabajo "El Entre". I Congreso Internacional de Promoción y Educación para la Salud. Organizado por Universidad Nacional de La Matanza, 26, 27 y 28 de noviembre. Buenos Aires.

Franco, T. y Merhy, E. (2011). El reconocimiento de la producción subjetiva del cuidado. Salud Colectiva, 7(1), 9-20.

Guelman, A. (2013). La vinculación universidad-sociedad como eje de la discusión prospectiva acerca del papel de la universidad. En Llomovatte, S.; Juarros, F. y Kantarovich, G. (Comps.). Reflexiones prospectivas sobre la universidad pública (pp. 99-109). Buenos Aires: Editorial de la Facultad de Filosofía y Letras, UBA.

Guldris, M.; Bang, C. y Barile, C. (2017). Una experiencia de voluntariado universitario en la facultad de psicología, creando lazos entre universidad y comunidad. Memorias IX Congreso Internacional de Investigación y Práctica Profesional en Psicología (Tomo I, pp. 127-129). Buenos Aires: Facultad de Psicología, UBA.

Lander, E. (2000). Colonialidad del saber, eurocentrismo y ciencias sociales. Buenos Aires: CLACSO/Unesco. Larrosa, J. (2003). La experiencia de la lectura: estudios sobre literatura y formación. México: Fondo de Cultura Económica.

Merhy, E. (2006). Salud: cartografía del trabajo vivo. Buenos Aires: Lugar Editorial.

Montero, M. (2003). Teoría y práctica de la psicología comunitaria. La tensión entre comunidad y sociedad. Buenos Aires: Paidós.

(2007). Introducción a la Psicología Comunitaria. Desarrollo, conceptos y procesos. Buenos Aires: Paidós Organización Panamericana de la Salud (OPS) (2009). Declaración de Lima sobre arte, salud y desarrollo. Lima: Editor.

Petz, I. (2015). Extensión universitaria: tendencias actuales y desafíos pendientes. Redes de Extensión, (1), 1-5. Ulloa, F.O. (1996). Novela clínica psicoanalítica. Historial de una práctica. Buenos Aires: Paidós.

Sousa Santos, B. de (1998). De la mano de Alicia: lo social y lo político en la posmodernidad. Santa Fé de Bogotá: Siglo del Hombre Editores.

Stolkiner, A.; Ardila, S. (2012). Conceptualizando la salud mental en las prácticas: consideraciones desde el pensamiento de la medicina social/salud colectiva latinoamericanas. Vertex Revista Argentina de Psiquiatría, XXIII(101), 52-56.

\section{Leyes}

Ley Nacional de Salud Mental, № 26.657 (BO: 03/12/2010). Derecho a la Protección de la Salud Mental. Extraído de http://www.msal.gov.ar/saludmental/images/stories/noticias/pdf/2013-09-26_ley-nacional-salud-mental.pdf 